\title{
Beschleunigung des Stoffaustausches von Gas-Flüssigkeits- Reaktionen durch Schallwellen am Beispiel der Fetthärtung
}

\author{
Achim Bartsch \\ Technische Universität Hamburg-Harburg, FSP 6-05, Eißendorfer Str. 38, 21073 Hamburg
}

Z. Naturforsch. 50a, 228-234 (1995); received November 14, 1994

Herrn Prof. Dr. Dr. h.c. Ewald Wicke gewidmet

Acceleration of mass transfer in gas/liquid-reactions by sonic vibrations:

fat hydrogenation as a test reaction

A method for increasing the mass transfer in gas/liquid-reactions by application of sonic vibration is described. The operating frequencies have been chosen such that the surfaces of the gas bubbles vibrate in resonance. At these operating frequencies (up to $1000 \mathrm{~Hz}$ ), the damping of sound waves by the bubbly liquid is low, which is important in large-scale applications. Hydrogenation of soybean oil in a bubble column has been carried out as a test reaction. An increase in mass transfer from dispersed bubbles to the liquid in terms of $k_{\mathrm{L}} a_{\mathrm{G}}$ of up to $36 \%$ has been effected by a relatively small amount of sonic power.

Key words: Mass transfer, Fat hydrogenation, Gas, Liquid, Sound, Surface waves.

\section{Einleitung}

Bei Gas-Flüssigkeits-Reaktionen ist der Stoffübergang des Gases in die Flüssigkeit meist der geschwindigkeitsbestimmende Schritt. Verbesserungen des Stoffaustausches werden normalerweise durch Reaktoreinbauten und/oder durch den Einsatz von Rührern erreicht. In der Vergangenheit hat man auch versucht, durch Anwendung von Schallwellen eine Erhöhung des Stoffübergangs zu bewirken. In der sog. "resonant bubble column" (s. z. B. [1, 2]) wird die gesamte GasFlüssigkeits-Säule zu Resonanzschwingungen angeregt, wodurch starke Stoffaustauschverbesserungen hervorgerufen werden können. Dieses Verfahren ist allerdings für industrielle Zwecke nicht geeignet, da man größere Reaktoren, d. h. hohe Gas-FlüssigkeitsSäulen, bei niedrigen Frequenzen $(<10 \mathrm{~Hz})$ und großen Amplituden ( $>0,5 \mathrm{~m}$ ) beschallen müßte, um nennenswerte Effekte auf den Stoffaustausch zu erzielen.

Ein zweiter Weg, der in der Vergangenheit eingeschlagen wurde, war der Einsatz von Ultraschall hoher Energie in Gas-Flüssigkeits-Systemen. So wurde u.a. versucht, die Hydrierung pflanzlicher Öle (Fetthärtung) mit Ultraschall hoher Energie zu beschleunigen $[3,4]$. Es wurden hohe Umsatzsteigerungen gemessen, die allerdings nicht auf eine Verbesserung des

Reprint requests to Dr. A. Bartsch.
Stoffaustausches zurückzuführen sind [5]. Eigene Untersuchungen haben ergeben, daß eine Erhöhung der Gesamtreaktionsgeschwindigkeit durch Ultraschallwellen hoher Energie auf eine Temperaturerhöhung des Reaktionsgemisches zurückzuführen ist. Vergleichende Messungen mit und ohne Einwirkung von Ultraschall hoher Energie zeigten bei strenger Einhaltung von isothermen Bedingungen keine Unterschiede in den Gesamtreaktionsgeschwindigkeiten. Da Reaktionsbedingungen vorlagen, bei denen der flüssigkeitsseitige Stofftransport die Reaktion kontrollierte, ergaben diese Versuche, ebenso wie in [5], daß der Stofftransport durch Ultraschall nicht wesentlich beeinflußt wird. Zusätzlich müssen zwei wesentliche Kritikpunkte angeführt werden, wenn man an einen Einsatz von Ultraschall in größeren Reaktoren denkt. Zum einen ist die pro Reaktionsvolumen eingesetzte Energie von einigen 100 Watt pro Liter für industrielle Zwecke viel zu hoch, und zum anderen wird Ultraschall in Gas-Flüssigkeits-Dispersionen sehr stark gedämpft $[6,7]$, so daß Ultraschall für eine Beschallung größerer Reaktoren nicht geeignet ist.

Dieser Arbeit liegt die Idee zugrunde, daß zur Erhöhung des Stoffaustausches gezielt die Gasbläschen selbst durch Schallwellen zum Schwingen angeregt werden sollen und so die Grenzfläche zwischen Gas und Flüssigkeit sowie die unmittelbare Umgebung der Gasbläschen intensiv bewegt werden. Es werden Bedingungen mitgeteilt, unter denen Schallwellen den 
Stoffaustausch von Gas-Flüssigkeits-Reaktionen beschleunigen.

\section{Grundlagen}

\subsection{Auswahl des Intensitäts- und Frequenzbereichs}

$\mathrm{Ob}$ ein Gasbläschen in einem Schallfeld aus einer es umgebenden Flüssigkeit Gas aufnimmt oder an sie abgibt, hängt wesentlich von Schalldruck und Frequenz der Schallwelle sowie von der Bläschengröße ab. Anhand von Abb. 1 soll verdeutlicht werden, wie sich Gasbläschen einer Gas-Flüssigkeits-Dispersion in einem Schallfeld verhalten. Näheres hierzu sowie die Berechnungsgrundlagen für die einzelnen Kurven findet man bei Neppiras [8]. Das Diagramm wurde berechnet für Wasserstoff-Bläschen (Druck: 1 bar) in mit Wasserstoff gesättigtem Sojaöl bei $180^{\circ} \mathrm{C}$ bei einer Anregungsfrequenz von $5000 \mathrm{~Hz}$. Übt man auf ein Gasbläschen mit einem Radius $r_{\mathrm{B}}$ einen so geringen Schallwechseldruck $P_{\mathrm{A}}$ aus, daß man im Gebiet der stabilen Kavitation bleibt (Punkt A in Abb. 1), so wird das Bläschen in der Unterdruckphase der Schallwelle größer und in der Überdruckphase kleiner. Die Schallwelle bewirkt dann also eine Volumenschwingung des Bläschens. Erhöht man den Schalldruck und überschreitet die Schwelle zur sog.

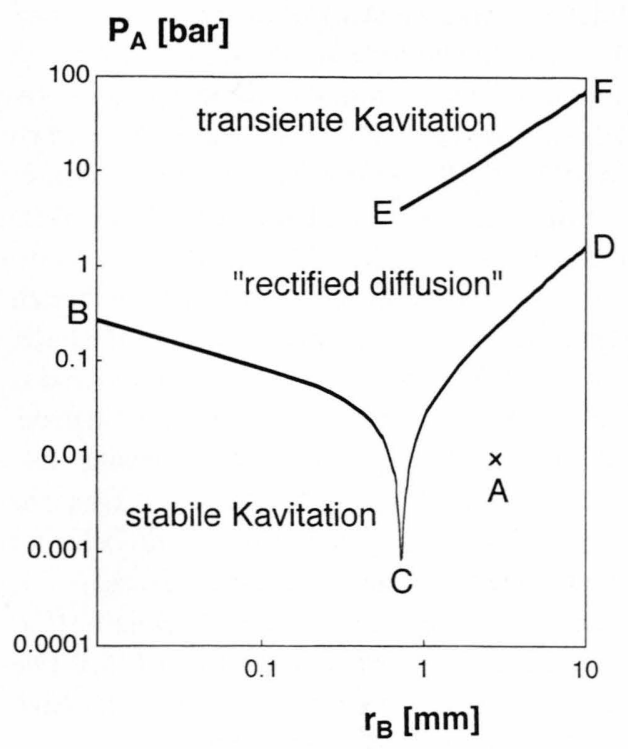

Abb. 1. Schwellenwerte des Schalldrucks zur "rectified diffusion" und zur transienten Kavitation in Abhängigkeit vom Bläschenradius $r_{\mathrm{B}}$. "rectified diffusion" (Linie B-C-D in Abb. 1), so nimmt das Gasbläschen Gas aus der Flüssigkeit auf und der mittlere Bläschenradius wird größer. Im Gebiet der "rectified diffusion" wird gearbeitet, wenn man eine Flüssigkeit entgasen will. Das Minimum der Kurve B-C-D liegt bei dem Bläschenradius, bei dem das Bläschen bei der hier angelegten Anregungsfrequenz von $5000 \mathrm{~Hz}$ die größte Amplitude der Volumenschwingung durchführt, d.h. die Anregungsfrequenz gleich der Resonanzfrequenz der Volumenschwingung ist.

Bei wesentlich höheren Schalldrücken (ab etwa 10 bar), tritt transiente Kavitation ein, d. h. innerhalb einer oder weniger Perioden wächst der Bläschenradius sehr stark an und das Bläschen kollabiert anschließend.

Zur Erhöhung des Stoffaustausches von Gas-Flüssigkeits-Reaktionen durch Anregung zur transienten Kavitation sind die erforderlichen Schalldrücke ab etwa 10 bar nur mit erheblichem Aufwand zu erreichen. Im Gebiet der "rectified diffusion" erzielt man das Gegenteil des gewünschten Effekts: die Flüssigkeit wird entgast. Der Schalldruck sollte also kleiner als der Schwellenwert des Schalldruckes zur "rectified diffusion" sein.

Bei der Auswahl einer geeigneten Frequenz zur Beschallung der Gas-Flüssigkeits-Dispersion muß in größeren Reaktoren berücksichtigt werden, daß eine Schallwelle im Frequenzbereich unterhalb der Resonanzfrequenz der Volumenschwingung der Gasbläschen nur wenig, bei und oberhalb dieser Frequenz jedoch sehr stark (Größenordnung $10 \mathrm{~dB} / \mathrm{cm}$ ) geschwächt wird (s. z. B. [6, 7]). Die Bläschenradien der meisten Gas-Flüssigkeits-Systeme liegen im Bereich von $0,5-2 \mathrm{~mm}$, die dazu gehörenden Resonanzfrequenzen im Bereich von 2-7 kHz. Während also Ultraschall wegen zu starker Schalldämpfung zur Beschallung größerer Reaktionsvolumina ungeeignet ist, sind Schallwellen mit Frequenzen im Bereich bis $2000 \mathrm{~Hz}$ in größeren Anlagen sinnvoll einzusetzen.

\subsection{Schwingungsformen von Gasbläschen in Flüssigkeiten}

Gasbläschen können in Flüssigkeiten Schwingungen der Moden $n=0,2,3,4, \ldots$ durchführen (s. Abbildung 2). Die Schwingungsmode $n=1$ kommt in der Natur nicht vor, da für eine solche Schwingung die rücktreibende Kraft fehlt. Die Resonanzfrequenz $v_{\text {res }}$ der Volumenschwingung (Mode $n=0$, Abb. 2) kann 


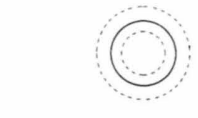

$\mathrm{n}=0$

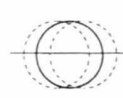

2

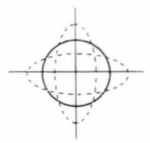

3

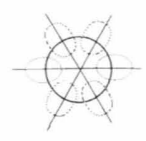

4

Abb. 2. Schwingungsformen (bzw. -Moden) von Gasbläschen.

bei hier in Frage kommenden Bläschengrößen nach der Minnaert-Formel ((1) in [9]) berechnet werden. Sie ist abhängig vom Bläschenradius $r_{\mathrm{B}}$, vom Verhältnis $\gamma$ der Wärmekapazitäten $C_{P} / C_{V}$ des Gases, von der Dichte der Flüssigkeit $\varrho_{\mathrm{L}}$ und vom Druck $P_{0}$ des Gases. Es gilt

$$
v_{\text {res }}=v_{0}=\frac{1}{2 \pi r_{\mathrm{B}}} \sqrt{\frac{3 \gamma P_{0}}{\varrho_{\mathrm{L}}}} .
$$

Alle anderen möglichen Schwingungsformen (Moden $n=2,3,4, \ldots$ in Abb. 2) sind Oberflächenschwingungen. Die Resonanzfrequenzen dieser Schwingungen sind nach Lamb [10] gegeben durch:

$$
v_{n}=\frac{1}{2 \pi r_{\mathrm{B}}} \sqrt{\frac{\left(n^{2}-1\right)(n+2) \sigma}{\varrho_{\mathrm{L}} r_{\mathrm{B}}}} .
$$

Sie hängen vom Schwingungsmode $n$, der Oberflächenspannung $\sigma$ sowie von $\varrho_{\mathrm{L}}$ und $r_{\mathrm{B}}$ ab.

Die Resonanzfrequenzen der niedrigen Schwingungsmoden ( $n=2$ bis 6 ) liegen für die meisten Gas/ Flüssigkeits-Systeme unterhalb von $1000 \mathrm{~Hz}$. Es wurde daher experimentell überprüft, ob sich durch Anregung der Oberflächenschwingungen der Gasbläschen der Stoffaustausch beschleunigen läßt.

\section{Experimenteller Teil}

Als Beispielreaktion wurde die Fetthärtung gewählt, weil sie über einen weiten Temperatur- und Druckbereich diffusionskontrolliert verläuft und die Meßbedingungen leicht und reproduzierbar einstellbar sind.

Der hierzu gebaute Schallreaktor (s. Abbildung 3) besteht aus einer normalen Blasensäule aus Glas (Innendurchmesser $46 \mathrm{~mm}$, Höhe $850 \mathrm{~mm}$ ), in die von unten Schall eingestrahlt wird. Der Schall wird erzeugt durch einen elektrodynamischen Baßlautsprecher (Gauß 4542), dessen Membran gegen eine aufgeschnittene und umgedrehte Aluminium-Dose

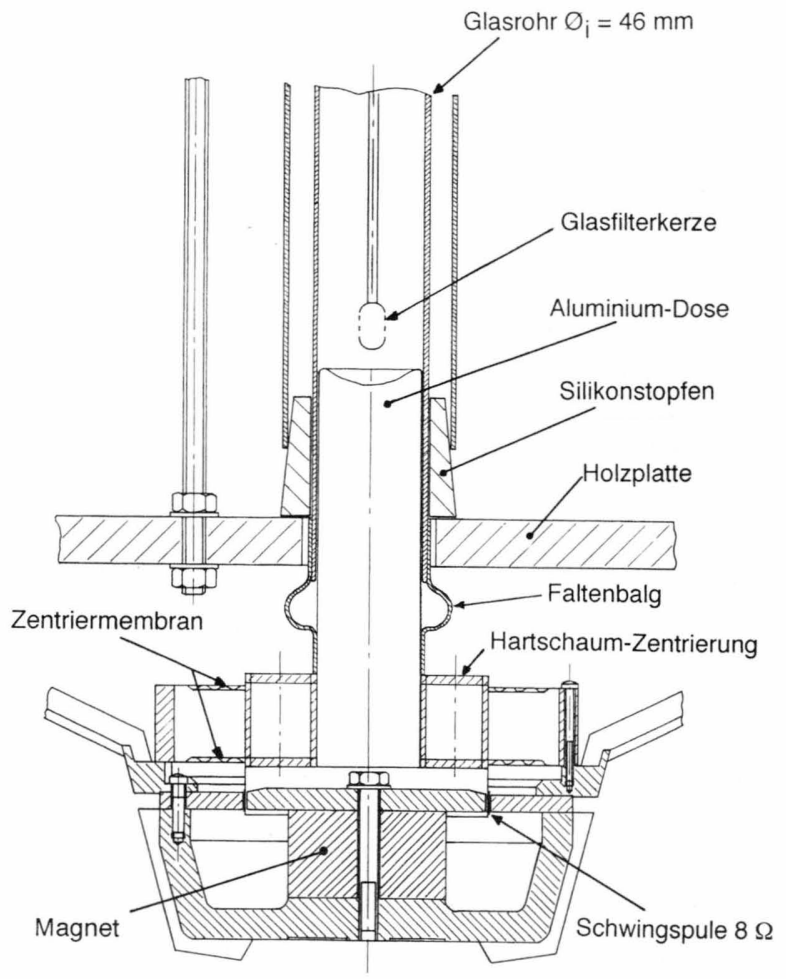

Abb. 3. Teilansicht des verwendeten Schallreaktors.

(handelsübliche Spraydose, Außendurchmesser $45 \mathrm{~mm}$ ) ausgetauscht wurde. Die Aluminium-Dose wird durch eine Hartschaum-Zentrierung, die in die Spulenhalterung geklebt wurde, gehalten. Ein Faltenbalg (Typ V6-67 SIMRIT ${ }^{\circledR}$ NBR, H. Reuss, Hamburg) dichtet zwischen Aluminium-Dose und Glasrohr. Der Wirkungsgrad dieses Systems, d. h. das Verhältnis von eingebrachter elektrischer Energie zur abgegebenen Schallenergie, ist sehr gering $(<1 \%)$, u. a. deshalb, weil die akustische Anpassung des Schallgebers an das Gas-Flüssigkeits-System noch nicht optimiert wurde.

Das Gas wird durch eine Filterkerze (Porosität D1, Schott, Mainz) in die Flüssigkeit eingeleitet. Das untere zugeschmolzene Ende der Filterkerze befindet sich etwa $1 \mathrm{~cm}$ oberhalb der Aluminium-Dose.

Das verwendete pflanzliche Öl was Sojaöl (Fa. HOBUM, Hamburg) mit einer Jodzahl von 135,1. Die Fettsäurenzusammensetzung ist aus Tab. 1 ersichtlich (Angaben der Fa. HOBUM, Hamburg).

Die Dichte des Sojaöls wurde pyknometrisch bestimmt. Die Meßdaten lassen sich im Temperaturbe- 
Tab. 1. Fettsäurenzusammensetzung des verwendeten Sojaöls.

C14 C16 C16:1 C18:0 C18:1 C18:2 C18:3 C20 C20:1 C22:0

\begin{tabular}{|c|c|c|c|c|c|}
\hline $\begin{array}{lll}\% & 0,1 & 10,6\end{array}$ & 0,1 & 3,6 & 20,5 & 55,7 & 8,7 \\
\hline
\end{tabular}

reich von $100^{\circ}-180^{\circ} \mathrm{C}$ durch die Geradengleichung

$$
\varrho_{\mathrm{L}}(\vartheta)=0,9307-\vartheta \cdot 6,576 \cdot 10^{-4} \quad[\mathrm{~g} / \mathrm{ml}]
$$

beschreiben (Temperatur $\vartheta$ in $\left[{ }^{\circ} \mathrm{C}\right]$ ).

Die Oberflächenspannung $\sigma_{\mathrm{L}}$ des Sojaöls wurde im gleichen Temperaturbereich mit einem Tensiometer (nach Lecomte du Noüy, Typ 8551, Fa. Krüss, Hamburg) bestimmt. Die Gleichung

$$
\sigma_{\mathrm{L}}(\vartheta)=36,1-\vartheta \cdot 0,0571 \quad[\mathrm{mN} / \mathrm{m}]
$$

gibt die gemessene Temperaturabhängigkeit wieder.

Bei den im folgenden beschriebenen Hydrierungen wurden jeweils $570 \mathrm{~g}$ Sojaöl am technischen NickelKatalysator (Typ G53, Südchemie, München) bei $180^{\circ} \mathrm{C}$ und 1 bar Gesamtdruck gehärtet. Die Strömungsgeschwindigkeit des Wasserstoffs (Reinheit 5,0) betrug $1 \mathrm{l} / \mathrm{min}$.

\section{Ergebnisse}

\subsection{Einflu $\beta$ von Schall auf den Umsatz}

In Abb. $4 \mathrm{a}$ ist am Beispiel der Hydrierung von Sojaöl (Katalysatorkonzentration $1 \mathrm{Gew}$ \%) die Umsatzsteigerung aufgetragen, die durch den Einsatz von Schall bei verschiedenen Frequenzen erreicht wurde. Die Nullinie entspricht also dem Umsatz an Wasserstoff ohne Schalleinwirkung. Dieser lag bei $45 \%$.

Das erhaltene Umsatzspektrum zeigt bei einigen Frequenzen Maxima, die durch Säulenresonanzschwingungen und durch Resonanzschwingungen der Bläschenoberfläche bzw. durch Kombination der beiden Schwingungsarten hervorgerufen werden. Die Maxima bei 52 und $94 \mathrm{~Hz}$ können, wie man während des Experimentes schon an einer intensiven Bewegung des Flüssigkeitsspiegels ausmachen konnte, Säulenresonanzen zugesprochen werden. Die Ausnutzung der Säulenresonanzen ist aus den in der Einleitung erwähnten Gründen für Anwendungen in größerem Maßstab nicht geeignet. Da die Resonanzfrequenz der Oberflächenschwingungen der Bläschen jedoch unabhängig von der Höhe der Gas-Flüssigkeits-Dispersion ist, eignet sich diese Bewegungsform für die industrielle Anwendung.

Um den Einfluß der Resonanzschwingungen der Säule und den der Resonanzschwingungen der Bläschenoberflächen auf den Umsatz zu erfassen, wurde von folgender Überlegung ausgegangen: Bei Anregung einer Säulenresonanz muß der Schallgeber relativ wenig Arbeit aufwenden, um einen hohen Schalldruck zu erzeugen. Bei frequenzunabhängigem Energieeintrag werden daher im Fall der Säulenresonanz die höchsten Schalldrücke gemessen. In Abb. 4 b sind nun die bei der Hydrierung gemessenen Schalldrücke in Abhängigkeit von der Frequenz aufgetragen. Ein Vergleich mit dem Umsatzspektrum (Abb. 4a) zeigt, daß offenbar auch das Umsatzmaximum bei $164 \mathrm{~Hz}$ durch Säulenresonanz verursacht wurde.

Bei Anregung von Schwingungen der Bläschenoberflächen schwingt die Oberfläche des Bläschens gegen die inkompressible Flüssigkeit. Je höher die Auslenkung, um so stärker nichtlinear schwingt die Oberfläche. Bei Resonanz ist die Auslenkung am größten, dann werden mithin verstärkt nichtlineare Anteile im Schallfeld gemessen. Im Fourier-Spektrum sind also im Resonanzfall der Oberflächenschwingungen die höheren Harmonischen stärker als sonst vertreten.

Als Maß für die Nichtlinearitäten - und damit auch für die Intensität der Schwingungen der Bläschenoberflächen - wird hier der in der Lautsprechertechnik häufig verwendete Klirrfaktor herangezogen. Der Klirrfaktor $K$ ist das Verhältnis der Schalldruckamplitudenquadrate der höheren Harmonischen $P_{n}$, $n=2,3, \ldots$ zu den Amplitudenquadraten aller Harmonischen $P_{n}, n=1,2, \ldots$. Es gilt daher:

$$
K=\sqrt{\frac{P_{2}^{2}+P_{3}^{2}+P_{4}^{2}+\ldots+P_{n}^{2}}{P_{1}^{2}+P_{2}^{2}+P_{3}^{2}+P_{4}^{2}+\ldots+P_{n}^{2}}} .
$$

Je höher der Klirrfaktor ist, um so stärker nichtlinear schwingt ein System. Da die Bläschenoberflächen bei Resonanz verstärkt nichtlinear schwingen, sind die Frequenzen, bei denen der Klirrfaktor ein Maximum hat, Resonanzschwingungen der Bläschenoberflächen zuzuordnen. In Abb. $4 \mathrm{c}$ ist der Klirrfaktor gegen die Frequenz aufgetragen. Vergleicht man diese Darstellung mit der Frequenzabhängigkeit der Umsatzsteigerung (s. Abb. 4 a), so wird deutlich, daß die Umsatzsteigerung von $50 \%$ bei $259 \mathrm{~Hz}$ sowie die Umsatzmaxima bei 223 und $141 \mathrm{~Hz}$ auf Resonanzschwingungen der Bläschenoberflächen zurückzuführen sind. 

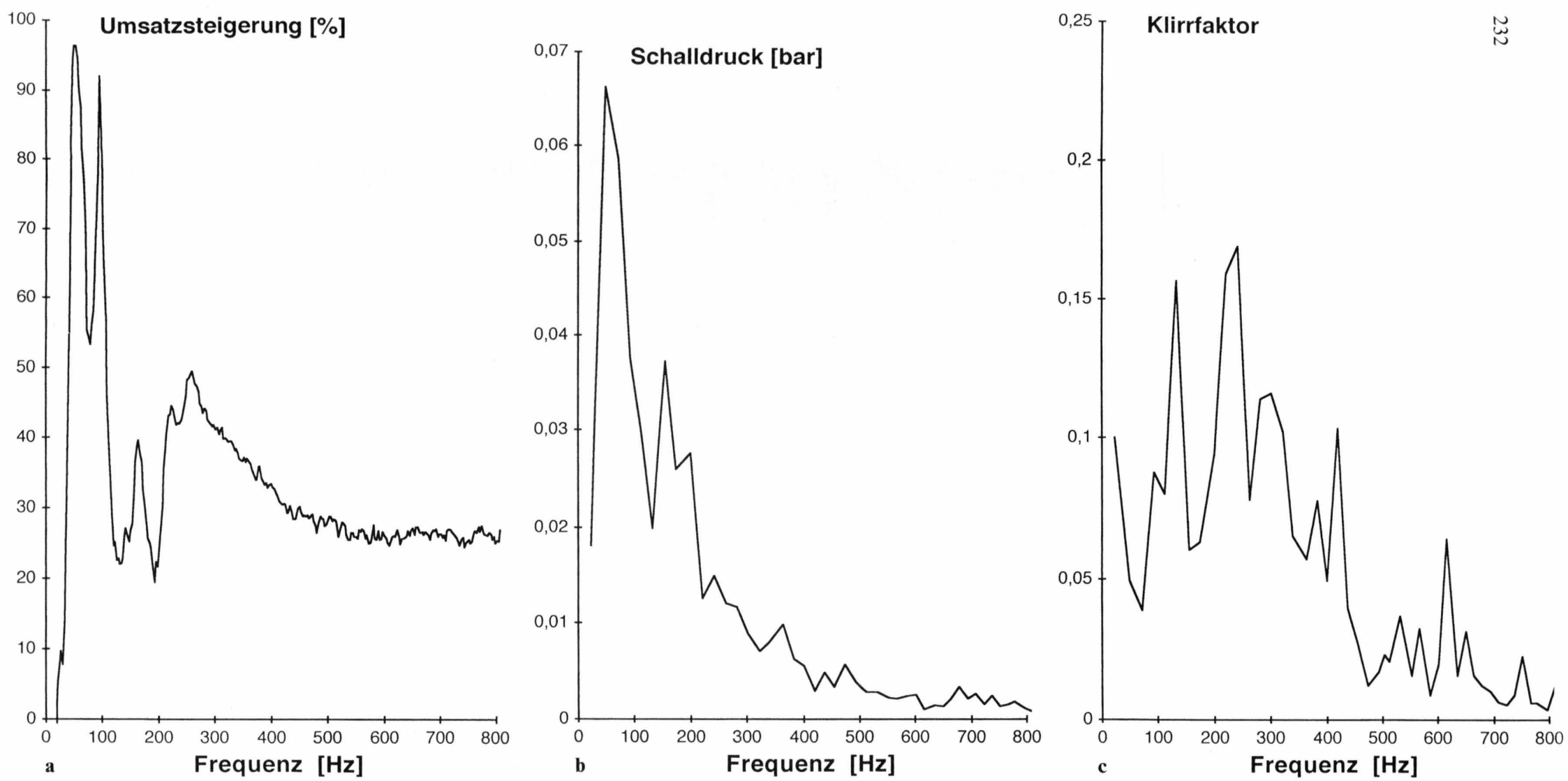

Abb. 4. Umsatzsteigerung durch Schall (a), Schalldruck (b) und Klirffaktor (c) bei der Härtung von Sojaöl in Abhängigkeit von der Anregungsfrequenz. 


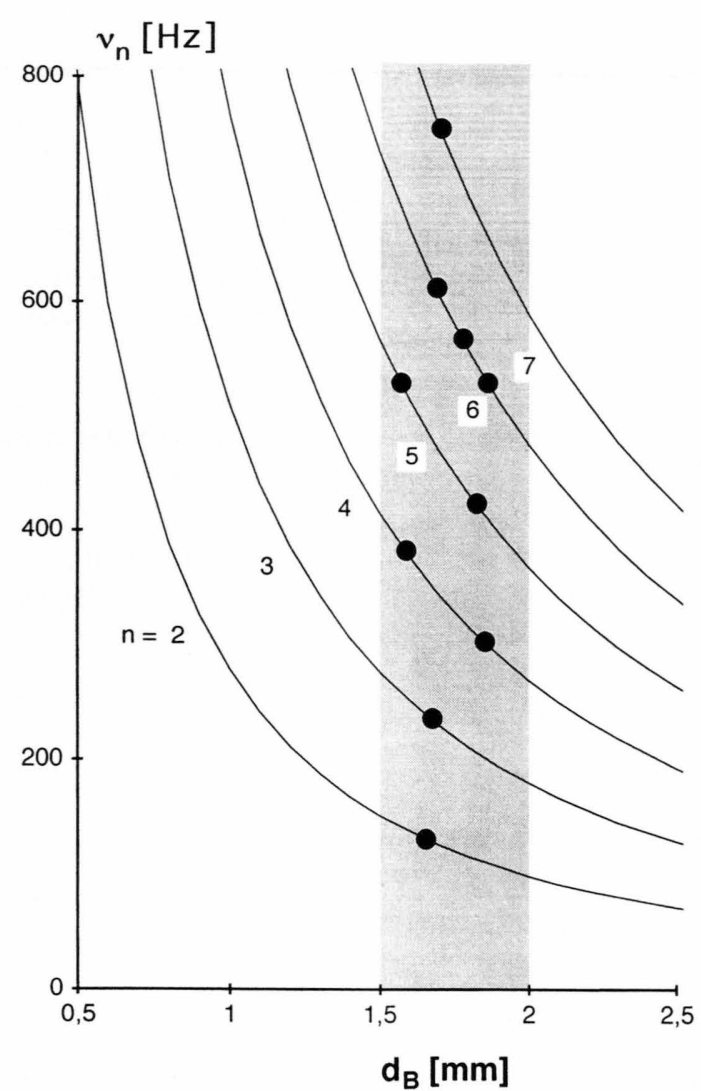

Abb. 5. Resonanzfrequenzen der Oberflächenschwingungen $n=2$ bis 7 in Abhängigkeit vom Bläschenradius $\left(\mathrm{H}_{2}\right.$ in Sojaöl, $180^{\circ} \mathrm{C}, 1$ bar) und Ermittlung von Bläschenradien mit Frequenzdaten aus Abbildung $4 \mathrm{c}$.

\subsection{Bestimmung von Bläschengrößen}

Den Frequenzen, bei denen Klirrfaktormaxima auftreten, lassen sich, wie in Abb. 5 gezeigt, Bläschendurchmesser zuordnen. $\mathrm{Zu}$ dieser Darstellung sind die Resonanzfrequenzen der einzelnen Oberflächenschwingungen für $n=2$ bis 7 als Funktion des Bläschendurchmessers aufgetragen, wie sie sich nach der Lamb-Formel für das System Wasserstoff in Sojaöl bei $180^{\circ} \mathrm{C}$ und 1 bar ergibt. Zur Bestimmung eines Bläschendurchmessers wurden die Frequenzbereiche, denen ein Klirrfaktormaximum zuzuordnen ist (s. Abb. $4 \mathrm{c}$ ), eingezeichnet. Er liegt zwischen 1,5 und $2 \mathrm{~mm}$. Eine grobe Abschätzung des Bläschendurchmessers durch Beobachtung sowie anhand theoretischer Überlegungen (s. z. B. [11]) bestätigen die Brauchbarkeit der hier vorgeschlagenen Bestimmungsmethode.

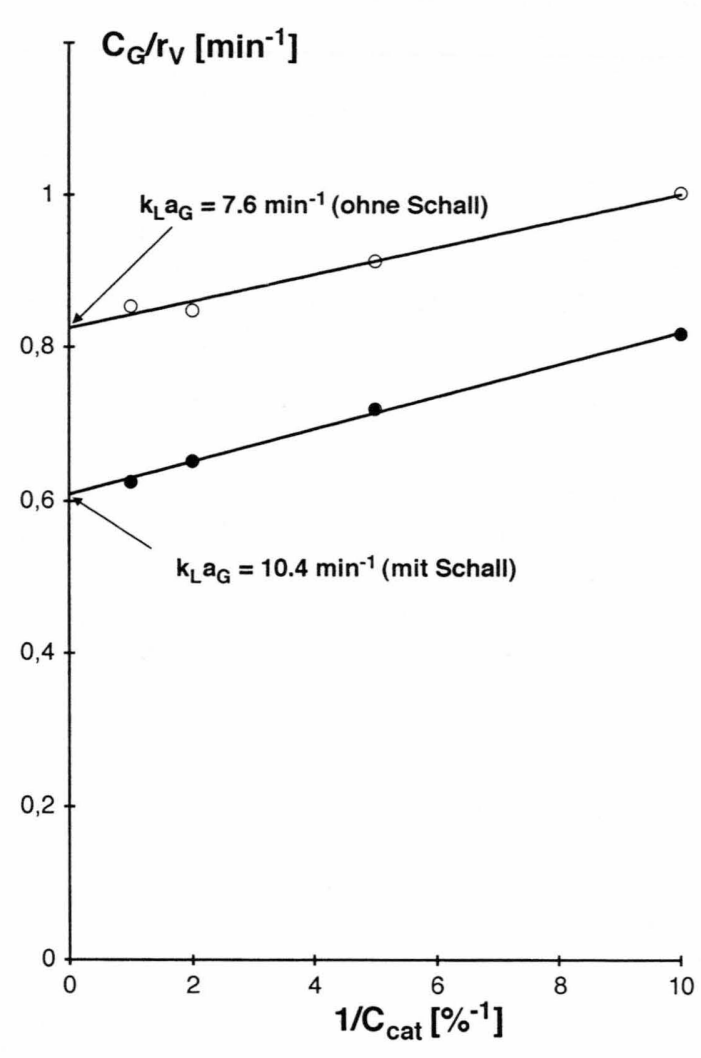

Abb. 6. Ermittlung von Stoffaustauschwerten aus Umsatzmessungen bei verschiedenen Katalysatorkonzentrationen mit und ohne Schalleinwirkung.

\subsection{Stoffaustauschmessungen}

Zur Bestimmung von Stoffaustauschwerten, d.h. von $k_{\mathrm{L}} a_{\mathrm{G}}$-Werten, wurden Umsatzmessungen unter den gleichen Reaktionsbedingungen wie oben, jedoch mit verschiedenen Katalysatorkonzentrationen durchgeführt.

Die Katalysatorkonzentrationen $C_{\text {cat }}$ wurden im Bereich von 0,1 bis $1 \%$ variiert. Es wurden Messungen mit Einwirkung von Schall, und zum Vergleich Messungen ohne Schalleinwirkung durchgeführt. Bei der gewählten Frequenz von $230 \pm 20 \mathrm{~Hz}$ sollten Oberflächenwellen, jedoch keine Säulenresonanzen angeregt werden.

Bei der Auswertung der Messungen wurde - wie allgemein üblich bei der Fetthärtung (s. z. B. $[12,13])$ davon ausgegangen, daß eine Reaktion 1. Ordnung vorliegt und der gasseitige Stofftransportwiderstand 
vernachlässigbar ist. Die gewünschten $k_{\mathrm{L}} a_{\mathrm{G}}$-Werte lassen sich dann bestimmen nach

$$
\frac{C_{\mathrm{G}}}{r_{\mathrm{V}}}=\frac{H}{k_{\mathrm{L}} a_{\mathrm{G}}}+\frac{A H}{C_{\text {cat }}}\left(\frac{1}{k_{\mathrm{C}}}+\frac{1}{k}\right) .
$$

$C_{\mathrm{G}} \quad H_{2}$-Konzentration in der Gasphase $\left[\mathrm{mol} / \mathrm{m}^{3}\right]$,

$r_{\mathrm{V}} \quad$ Reaktionsgeschwindigkeit $\left[\mathrm{mol} /\left(\mathrm{m}^{3} \cdot \mathrm{min}\right)\right]$

$k_{\mathrm{L}} \quad$ Stofftransportkoeffizient (flüssigkeitsseitig) [m/min],

$k_{\mathrm{C}} \quad$ Stofftransportkoeffizient am Katalysator [m/min],

$a_{\mathrm{G}} \quad$ Oberfläche der Gasblasen $\left[\mathrm{m}^{2} / \mathrm{m}^{3}\right]$,

$H \quad \mathrm{H}_{2}$-Löslichkeit $\left[\left\{\mathrm{mol} / \mathrm{m}^{3}\right.\right.$ (Gasphase) $\} /\left\{\mathrm{mol} / \mathrm{m}^{3}\right)(\mathrm{O} 1$ Phase)\}],

$a_{\text {cat }} \quad$ Katalysatoroberfläche $\left[\mathrm{m}^{2} / \mathrm{m}^{3}\right]$,

$C_{\text {cat }} \quad$ Katalysatorkonzentration [\%],

$A$ Proportionalitätskonst. zw. $a_{\text {cat }}$ und $C_{\text {cat }}\left[\left(\mathrm{m}^{3} \cdot \%\right) / \mathrm{m}^{2}\right]$

Trägt man, wie in Abb. 6 geschehen, $C_{\text {gas }} / r_{\mathrm{v}}$ gegen $1 / C_{\text {cat }}$ auf, so erhält man als Ordinatenabschnitt den
Wert für $H / k_{\mathrm{L}} a_{\mathrm{G}}$. Mit einer $\mathrm{H}_{2}$-Löslichkeit von 6,27 (geschätzt nach Werten aus [14]) erhält man für die Messung ohne Schalleinwirkung einen $k_{\mathrm{L}} a_{\mathrm{G}}$-Wert von 7,6 $\mathrm{min}^{-1}$. Mit Schalleinwirkung ergibt sich ein $k_{\mathrm{L}} a_{\mathrm{G}}$-Wert von $10,4 \mathrm{~min}^{-1}$. Zusätzlich läßt sich aus Abb. 6 erkennen, da $\beta$ das Geschehen am Katalysator selbst durch die Schalleinwirkung nicht beeinflußt wird, da die Steigungen und somit auch die $k_{\mathrm{C}^{-}}$und die $k$-Werte in etwa gleich sind.

Die Beschleunigung des Stofftransports durch Schalleinwirkung beträgt $36 \%$ bei einer eingebrachten Schalleistung von wenigen Milliwatt. Der geringe Aufwand an Schallenergie wird darauf zurückgeführt, daß durch Schalleinwirkung die Bläschen gezielt zum Schwingen angeregt werden können, wobei energiegünstig Resonanzeffekte auszunutzen sind, die im gerührten Reaktor nicht zur Verfügung stehen.
[1] G. J. Jameson, Trans. Instn. Chem. Engrs. 44, T91 (1966).

[2] M. H. I. Baird, Chem. Eng. Sci. 18, 685 (1963).

[3] K. J. Moulton, S. Koritala, and E. N. Frankel, JAOCS 60, 1257 (1983).

[4] P. J. Wan, M.wa Muanda, and J. E. Covey, JAOCS 69, 876 (1992).

[5] S. V. Jadhav and V. G. Pangarkar, JAOCS 66, 362 (1989).

[6] E. Silberman, J. Acoust. Soc. Amer. 29, 925 (1957).

[7] A Prosperetti, Appl. Sci. Res. 38, 145 (1982).

[8] E. A. Neppiras, Ultrasonics 18, 201 (1980).
[9] M. Minnaert, Phil. Mag. (Ser. 7) 16, 253 (1933).

[10] H. Lamb, Hydrodynamics, Cambridge University Press, 6th. ed., (1975), S. 475.

[11] K. Akita and F. Yoshida, Ind. Eng. Chem. Proc. Des. Dev. 13, 84 (1974).

[12] J. M. Smith, Chemical Engineering Kinetics, McGrawHill Book Co., Singapore u. a. 3rd. ed. 1981, p. 432.

[13] J. W. E. Coenen, in J. H. de Boer (ed.), The Mechanism of Heterogeneous Catalysis, S. 126, Elsevier Publishing Company, New York, 1960.

[14] L. Bern, M. Hell, and N.-H. Schöön, JAOCS 52, 182 (1975). 